\title{
Postgenomic chemical biology
}

\section{Chemical biology contributions will become increasingly important as we enter the second decade of the postgenomic era.}

\begin{abstract}
On 26 June 2000, the leaders of the international Human Genome Project and Celera Genomics joined U.S. President Bill Clinton at the White House to herald the arrival of the genomic era. The event was accompanied by predictions of an era of personalized medicine in which new diagnostics would reveal genetic sequence information that would in turn inform medical decisions involving the use of targeted therapeutic agents. In the scientific community, some believed that DNA sequence data would open 'the book of life' and lead to the swift resolution of biology's remaining puzzles.
\end{abstract}

Ten years on, these euphoric forecasts have not yet been realized. However, the availability of genomic data has revolutionized how scientists, including chemical biologists, understand and explore biological systems (Nature 465, 1000-1001, 2010). Though many biological researchers make use of primary gene sequences on a daily basis, it is clear that most of the answers to biology's puzzles are found not in the sequences themselves but in linking 'functional genomics' - the assignment of gene function and interactions-directly to the systems of biomolecules that mediate these functions in cells: proteins, small RNAs, carbohydrates, lipids and small molecules. Chemical biologists, who have grown up in this genome-rich milieu, are particularly well placed to contribute to postgenomic knowledge in various ways, including developing new technologies, expanding our understanding of small molecules in biology and uncovering the molecular mechanisms of biological pathways.

Chemical biology has provided essential tools that enable researchers to acquire and interpret genome- and systems-wide biomolecular data. For example, the development of new DNA sequencing technologies has supported the decoding of genomes from diverse sources-including model organisms and human pathogenswith unparalleled speed and accuracy. In the past 10 years, chemical tools such as mass spectrometry, activity-based probes and molecular arrays have provided direct approaches for profiling the cellular abundance and functions of nucleic acids, proteins, lipids and carbohydrates. It is clear that many more chemical tools, along with expanded computational and bioinformatic approaches, will be needed to obtain and integrate future 'omic data sets, moving toward the ultimate goal of linking genetic sequence and biological function.

A central aim of chemical biology research-understanding the identities and roles of small molecules in cells-has been bolstered by the availability of genomic information. For instance, DNA sequencing data has already influenced how chemical biologists discover new natural products and biosynthetic pathways. In contrast to traditional isolation approaches, most natural product studies now begin with bioinformatic analysis of biosynthetic gene clusters from DNA sequence data. Genomic studies have also initiated a resurgence of interest in metabolic pathways, which continues to spur new metabolomic approaches for profiling the distribution and functions of small molecules within cells.

Increasingly, genetic information will support chemical biologists as they seek to identify new chemical probes for dissecting biological pathways (Curr. Opin. Chem. Biol. 7, 511-515, 2003). In much the same way that genome sequencing efforts in Saccharomyces cerevisiae led to genetic knockout strains for exploring yeast biology, decoding of the human genome has stimulated the search for chemical probes that reversibly modulate the function of each human gene (Nat. Chem. Biol. 1, 64-66, 2005). Not only will genetic information help direct probe development efforts toward the pathways of most significant biological interest, it will guide the design of chemical libraries and high-throughput screening strategies that will make it possible for academic laboratories to identify new chemical probes for any target of choice.

With this grounding in small-molecule chemistry, chemical biology is also poised to contribute significantly to next-generation drug discovery efforts. Shortly after the publication of the draft human genome, Hopkins and Groom (Nat. Rev. Drug Disc. 9, $727-730,2002)$ highlighted the many challenges and opportunities for expanding the 'druggable genome'. Chemical biologists are uniquely equipped to take advantage of these opportunities by expanding 'chemical space, identifying and optimizing active small molecules and developing new methods for validating the cellular targets and specificity of active compounds. In addition, chemical biology studies have identified new modulators of targets that were previously thought to be 'undruggable' - such as protein-protein interactions (Nature $\mathbf{4 5 0}$, 1001-1009, 2007) — and have forced ongoing re-examinations of the traditional models of drug design (Nat. Chem. Biol. 4, 682-690, 2008) and focused attention on the potential utility of designed polypharmacology (Nat. Chem. Biol. 4, 635-638, 2008; Nat. Chem. Biol. 4, 691-699, 2008). Although analyses such as the International HapMap Project (http://hapmap.ncbi.nlm.nih.gov/) and genome-wide association studies offer perspectives into the genetics of human disease, modern drug discovery efforts need to be grounded in molecular insights and knowledge of disease biology mechanisms, which are areas of strength for chemical biology investigation.

Genomic science has made significant contributions in the past decade, but it also has highlighted the limits of our current knowledge of biology. For example, data showing that the genome is actively transcribed even in domains that do not code for proteins have reformulated our concept of a gene. Genomic information has shown that the regulation of gene expression is even more complex than previously thought: diverse noncoding RNAs transcribed from regions that had been termed 'junk DNA' and epigenetic modifications of DNA and chromatin proteins are essential mechanisms for modulating gene expression in organisms. Chemical biology can move these studies toward molecular-level understanding and bridge the mechanistic gaps in our knowledge. In turn, chemical understanding opens potential avenues for the design and optimization of biological systems for synthetic biology applications.

Though many of the predicted biomedical advances are only just beginning to appear, the elucidation of the human genome has already spurred biological research forward at a remarkable pace. The flood of new data and discoveries from genomics and other 'omic sciences offers unparalleled opportunities for researchers committed to understanding and manipulating biological systems at the molecular level. Chemical biologists have already met this challenge, and we look forward to the next 10 years of chemical biology in the postgenomic era. 